\title{
Adenine Inhibition of the Rate of Sorocarp Formation in Dictyostelium discoideum
}

\author{
BY M. I. KRICHEVSKY AND L. L. LOVE \\ U.S. Department of Health, Education, and Welfare, Public Health \\ Service, National Institutes of Health, National Institute of \\ Dental Research, Bethesda, Maryland 20014, U.S.A.
}

(Received 29 July 1964)

\section{SUMMARY}

Some characteristics of adenine inhibition of the rate of sorocarp formation by Dictyostelium discoideum are described. As little as $1.25 \times 10^{-3} \mathrm{M}$ adenine was markedly inhibitory. All of the stimulants tested (histidine, glucose, $\mathrm{Mg}^{2+}, \mathrm{K}^{+}, \mathrm{PO}_{4}^{8-}, \mathrm{Na}^{+}$) were capable of stimulating the rate of fructification even in the presence of adenine, although to a lesser extent. It is concluded that the inhibition by adenine and the stimulation by the other materials listed are independent phenomena.

\section{INTRODUCTION}

The ability of a variety of materials to stimulate the rate of development in Dictyostelium discoideum was described previously (Krichevsky \& Wright, 1963; Krichevsky \& Love, 1964). In connexion with this work it was decided to investigate the phenomenon of adenine inhibition of morphogenesis as reported by Bradley, Sussman \& Ennis (1956). The present report further characterizes the inhibition of development by adenine.

\section{METHODS}

The procedures for growing and harvesting the myxamoebae, as well as for determining the effect of materials on the rate of morphogenesis, were as described previously (Krichevsky \& Wright, 1963). The usual method was to incubate all test plates and a control plate containing plain $2.5 \%$ agar until the control plate was about to finish forming its first complete sorocarp. If one waits long enough, all plates would contain approximately equal numbers of mature sorocarps. Thus, the number of sorocarps at the time of counting is a measure of the stimulation of the relative rate of sorocarp formation in the presence of the test substance as compared with the water control. However, in determining adenine inhibition, the control plate containing only water was allowed to achieve almost complete fructification before comparative counts were made.

\section{RESULTS}

As reported by Bradley et al. (1956) adenine is capable of inhibiting the rate of aggregation when compared to water controls. When the criterion of measurement of the inhibitory capacity of adenine was that of comparative rate of sorocarp formation, the results shown in Fig. 1 were obtained. The degree of inhibition was approximately proportional to adenine concentration at the lower concentrations.

The effect of adenine on the rate of sorocarp formation in the presence of $0.02 \mathrm{M}$ - 


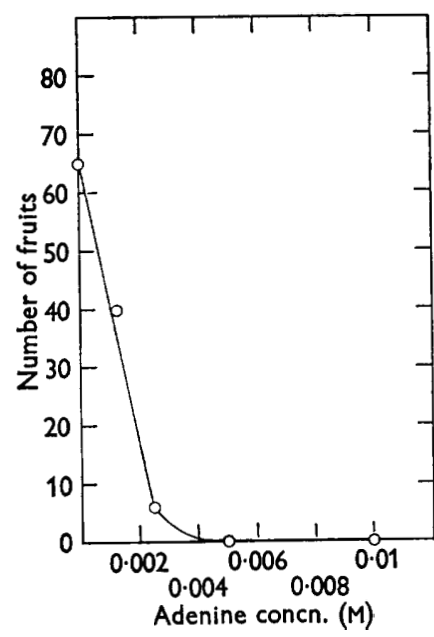

Fig. 1

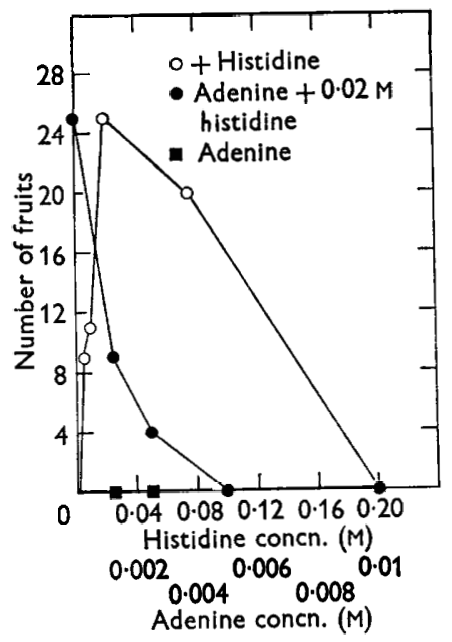

Fig. 2

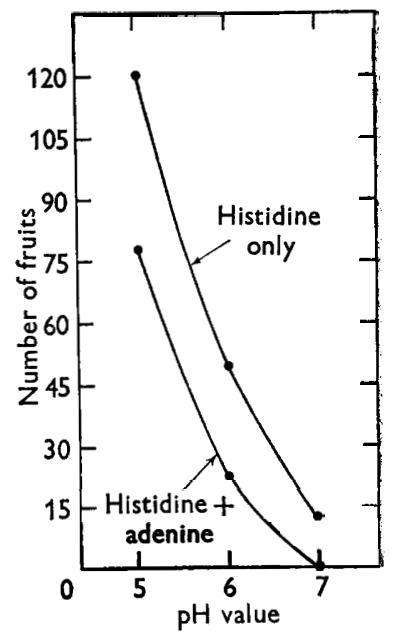

Fig. 3

Fig. 1. Inhibition of sorocarp of Dictyostelium discoideum formation by adenine. The medium was adjusted to $\mathrm{pH} 6 \cdot 2$ in all cases.

Fig. 2. The effect of adenine on sorocarp formation of Dictyostelium discoideum in the presence of L-histidine. All samples were at $\mathrm{pH} \mathrm{6 \cdot 2}$. The L-histidine final concentration was $0.02 \mathrm{M}$ when used with the indicated concentrations of adenine. Three $0.01 \mathrm{ml}$. drops of amoebal suspension were added per test Petri dish instead of the usual six drops.

Fig. 3. The effect of histidine + adenine at various $\mathrm{pH}$ values on sorocarp formation by Dictyostelium discoideum. The final adenine concentration was $4 \times 10^{-3} \mathrm{M}$; that of the L-histidine was $4 \times 10^{-2} \mathrm{M}$.

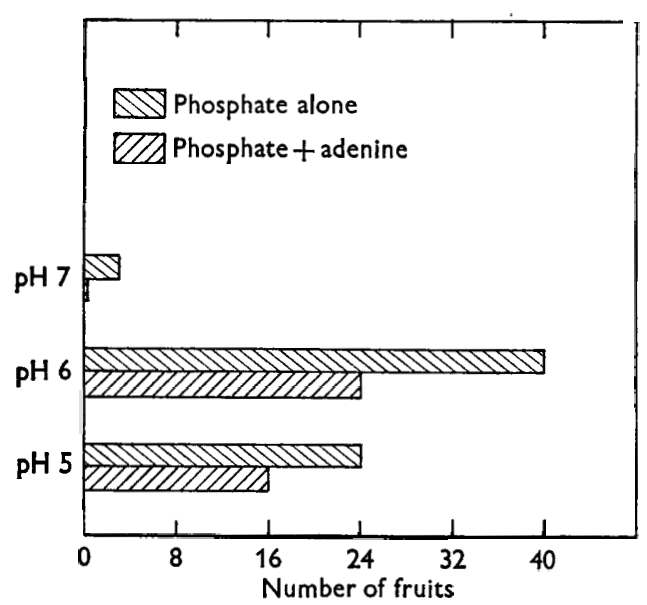

Fig. 4

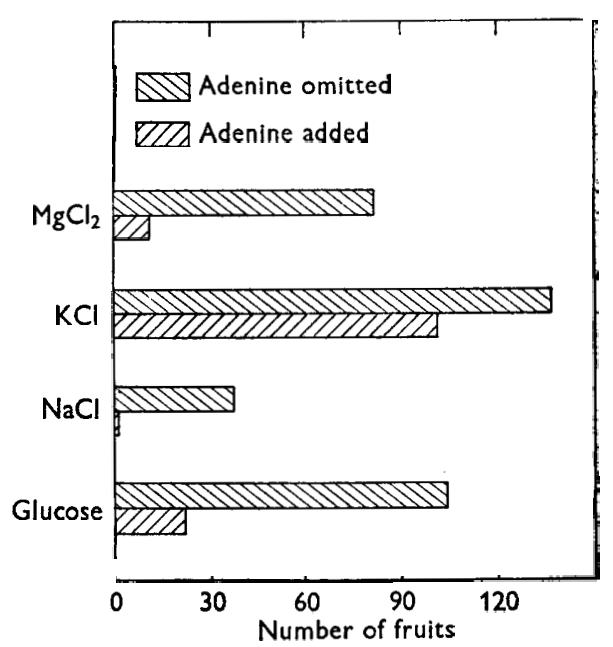

Fig. 5

Fig. 4. The effect of phosphate and adenine combinations at various pH values on sorocarp formation by Dictyostelium discoideum. The final concentration of adenine was $4 \times 10^{-3} \mathrm{M}$. Sodium phosphate buffers were added to a final phosphate concentration of $10^{-2} \mathrm{M}$, at the $\mathrm{pH}$ values indicated.

Fig. 5. Sorocarp formation by Dictyostelium discoideum in the presence of adenine and various stimulants. Where indicated, additions were made to final concentrations as follows : Adenine, $4 \times 10^{-3} \mathrm{M}$; glucose, $0.05 \mathrm{M} ; \mathrm{NaCl}, 0.03 \mathrm{M} ; \mathrm{KCl}, 0.03 \mathrm{M} ; \mathrm{MgCl}_{2}, 0.015 \mathrm{M}$. 
histidine ( $\mathrm{pH} \mathrm{6.2)} \mathrm{is} \mathrm{illustrated} \mathrm{in} \mathrm{Fig.} \mathrm{2.} \mathrm{It} \mathrm{may} \mathrm{be} \mathrm{seen} \mathrm{that} \mathrm{the} \mathrm{variation} \mathrm{in} \mathrm{the}$ rate of sorocarp formation with concentration of adenine was approximately the same regardless of the presence or absence of histidine (compare with Fig. 1). The concentration curve for histidine alone is presented for comparison.

It was found (Fig. 3) that, in the presence of $0.04 \mathrm{~m}$-histidine, adenine had approximately the same relative inhibitory effect regardless of the $\mathrm{pH}$ value of the medium. As described previously (Krichevsky \& Wright, 1963), histidine stimulated in inverse relationship to the $\mathrm{pH}$ value. In contrast, no relationship was observed which could be ascribed to the $\mathrm{pH}$ value of the adenine solution. This last observation is further illustrated (Fig. 4) by the effect of sodium phosphate buffer and adenine. The difference in sorocarp formation between those samples containing adenine and those without was about the same at $\mathrm{pH} 5$ and $\mathrm{pH} 6$. Since sodium phosphate was only slightly stimulatory at $\mathrm{pH} 7$ (in contrast to the same concentrations of potassium phosphate at $\mathrm{pH} 7$ as reported previously; Krichevsky \& Wright, 1963) it was not possible to make the same test at this $\mathrm{pH}$ value.

The effect of adenine with other stimulants of morphogenesis is shown in Fig. 5. Those compounds which best stimulated the rate of fructification (i.e. glucose, KCl, $\mathrm{MgCl}_{2}$ ) were able to do so even in the presence of adenine. In contrast, $\mathrm{NaCl}$, which was a poor stimulant alone (Krichevsky \& Wright, 1963) did not stimulate to any discernible extent in combination with adenine.

\section{DISCUSSION}

In the main, materials which had been found to stimulate sorocarp formation in Dictyostelium discoideum did so most efficiently at concentrations greater than $\mathbf{0} \cdot \mathbf{1} \mathrm{M}$. In contrast, adenine was markedly inhibitory at much lower concentrations. None of the materials tested in presence of adenine appeared capable of modifying the effect of adenine. That is, the rate of sorocarp formation was less in the presence of adenine than without adenine. This result was qualitatively similar, irrespective of the other components present in the test system. Since no interaction was noted between adenine inhibition of the rate of sorocarp formation and stimulation of it by those materials tested (with the possible exception of $\mathbf{K}^{+}$), it would appear that the mechanisms by which the inhibition and stimulation are effected differ from one another. That is, both adenine inhibition and metabolite stimulation are observed simultaneously. It was suggested previously (Krichevsky \& Love, 1964) that histidine stimulated by changing the permeability of the amoebae. It has been observed that histidine and other stimulants do indeed modify the exit of some macromolecular materials (namely ribonucleic acid, protein) from cells (Krichevsky \& Love, unpublished data). Thus it seems unlikely that the mechanism of adenine inhibition involves changing the permeability of the developing amoebae.

\section{REFERENCES}

Bradley, S. G., Sussman, M. \& Ennis, H. L. (1956). Environmental factors affecting the aggregation of the cellular slime mold, Dictyostelium discoideum. J. Protozool. 3, 33.

KrICHevsky, M. I. \& Love, L. L. (1964). The uptake and utilization of histidine by washed amoebae in the course of development in Dictyostelium discoideum. J. gen. Microbiol. 34, 483.

Krichevsky, M. I. \& Wright, B. E. (1963). Environmental control of the course of development in Dictyostelium discoideum. J. gen. Microbiol. 32, 195. 